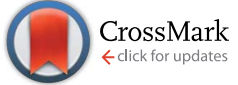

Cite this: J. Mater. Chem. A, 2015, 3, 12890

Received 31st March 2015 Accepted 19th May 2015

DOI: $10.1039 / \mathrm{c} 5 \mathrm{ta0} 2354 \mathrm{~h}$

www.rsc.org/MaterialsA

\section{High-silica, heulandite-type zeolites prepared by direct synthesis and topotactic condensation $\dagger$}

\begin{abstract}
Joel E. Schmidt, ${ }^{a}$ Dan $\mathrm{Xie}^{\mathrm{b}}$ and Mark E. Davis ${ }^{* a}$
There are both natural minerals and synthetic zeolites that possess the HEU framework topology. These materials have a limited compositional range ( $\mathrm{Si} / \mathrm{Al}<6$ ), and the natural zeolites often contain a large amount of impurities such as $\mathrm{Fe}^{3+}$. The preparation of impurity-free HEU-type zeolites with higher Si/Al ratio could open many areas of application, particularly in catalysis. Here, we report the first high-silica HEU-type zeolite that can be prepared via two different procedures. In the first method high-silica HEU (denoted CIT-8) is prepared using a topotactic condensation mechanism (layered precursor denoted CIT-8P); CIT-8P is obtained from a low-water synthesis in fluoride media. CIT-8 prepared in this manner has a product $\mathrm{Si} / \mathrm{Al}$ ratio of $9.8 \pm 0.7$ and a micropore volume of $0.10 \mathrm{~cm}^{3} \mathrm{~g}^{-1}$ (measured by nitrogen adsorption). The variable temperature powder X-ray diffraction shows that CIT- 8 forms via topotactic condensation from CIT-8P along the $b$ axis. Additionally, high-silica heulandite can be synthesized directly from a hydroxide-mediated reaction mixture (denoted $\mathrm{CIT}-8 \mathrm{H}$ ), and has a Si/Al ratio of $6.4 \pm$ 0.3 and a micropore volume of $0.10 \mathrm{~cm}^{3} \mathrm{~g}^{-1}$. Both synthesis methods produce zeolites that expand the compositional range of HEU-type zeolites. These synthetic methods allow for the addition of other heteroatoms, and titanium-containing $\mathrm{CIT}-8$ is prepared as an illustrative example.
\end{abstract}

\section{Introduction}

Natural zeolites form under hydrothermal processes, normally near volcanic deposits, and according to the International Mineralogical Association definition, there were known to be 93 different mineral species belonging to 44 different framework types as of $2014 .^{1}$ Approximately 2.5 million metric tons of natural zeolites are consumed per year, compared to 1.7-2 million metric tons of synthetic zeolites. ${ }^{2}$ Natural zeolites are employed in applications such as separations, impurity removal (such as ammonia), and as additives in paper, consumer products and animal feed. ${ }^{3}$ Many natural zeolites are known to have synthetic analogs that can be produced at different compositions than the natural material. Additionally, natural zeolites often have large amounts of impurities, making them unsuitable for many applications. Natural zeolites commonly occur at compositions (typically low $\mathrm{Si} / \mathrm{Al}$ ratio, e.g., below 5) that are not thermally or hydrothermally stable, further rendering them unusable in demanding process conditions, such as catalytic applications. Therefore, discovery of synthetic methods to produce analogs of natural zeolites that result in desired frameworks free of impurities and exhibiting robust

\footnotetext{
${ }^{a}$ Chemical Engineering, California Institute of Technology, Pasadena, CA 91125, USA. E-mail: mdavis@cheme.caltech.edu

${ }^{b}$ Chevron Energy Technology Company, Richmond, CA 94802, USA

$\dagger$ Electronic supplementary information (ESI) available: Deconvolution of ${ }^{29} \mathrm{Si}$ NMR spectrum and surface silanol calculation. See DOI: 10.1039/c5ta02354h
}

thermal and hydrothermal stability (typically Si/Al ratio higher than 5) are highly desired. One recent example of this approach is that of the synthetic analogue of the mineral boggsite $\left(\mathbf{B O G}^{4}\right)$, recently prepared as ITQ-47. ${ }^{5}$ The BOG framework has a 2-dimensional system of intersecting 10- and 12-membered rings (MRs) and was prepared synthetically using an organic structure directing agent based on phosphazenes, as a silicoborate framework material instead of the aluminosilicate material that is the rare natural zeolite. ITQ-47 has been evaluated for catalytic applications including the production of cumene by the alkylation of benzene with propylene where it shows better product selectivities to cumene than *BEA, the current industrial standard material for this reaction. ${ }^{5}$

Zeolites with the HEU framework exist as both natural minerals as well as their synthetic analogs. The natural materials can be excavated from quarries at low cost, and are used in applications including building materials, paper fillers, livestock feed supplements, water purification, dehydration and gas separations. ${ }^{3}$ The HEU framework contains a pore channel system with openings consisting of $10 \mathrm{MRs}$ in the [001] direction that are $3.1 \times 7.5 \AA$ as well as 8 MRs with dimensions of $3.6 \times 4.6 \AA$ in the [001] direction. Additionally, there is another set of 8 MRs along the [100] direction with dimensions of $2.8 \times$ $4.7 \AA{ }^{6}$ Materials with the HEU framework are divided into two distinct classes based on $\mathrm{Si} / \mathrm{Al}$ ratio. Those with $\mathrm{Si} / \mathrm{Al}$ of less than 4 are known as heulandite and those with $\mathrm{Si} / \mathrm{Al}$ greater than 4 are known as clinoptilolite, or silica-rich heulandite. The key difference in these materials is those with $\mathrm{Si} / \mathrm{Al}$ of less than 4 are 
not thermally stable to calcination above $350{ }^{\circ} \mathrm{C}$ (though the literature contains some ambiguity about the definition). ${ }^{7}$ Natural heulandites are often encountered containing $\mathrm{Ba}, \mathrm{Ca}$, $\mathrm{Na}, \mathrm{K}$ and $\mathrm{Sr}$ in addition to a significant amount of iron as $\mathrm{Fe}^{3+}$ (up to $1.5 \%$ of the tetrahedra). ${ }^{3}$

A synthetic method to produce a high-silica heulandite was first reported by removing aluminum from natural clinoptilolite to make a material with $\mathrm{Si} / \mathrm{Al} \geq 5.5$, known as LZ-219. ${ }^{8}$ Later, methods were developed to produce synthetic heulandites across a range of $\mathrm{Si} / \mathrm{Al}=2.5-6$ using various sources of silica and alumina and a wide range of inorganic cations ( $\mathrm{Ca}, \mathrm{K}, \mathrm{Li}, \mathrm{Na}, \mathrm{Ca}$, $\mathrm{Sr}$ ) under hydrothermal synthesis conditions., ${ }^{7,9-12}$ These materials have been considered for applications such as gas cleaning and separations, ion exchange, isomerization of 1-butene and xylene, methanol dehydration, acetylene hydration and the separation of nitrogen from natural gas., ${ }^{7,13}$ In any of these applications, the ability to tailor the framework composition is important for material properties such as exchange capacity, thermal and hydrothermal stability as well as pore accessibility.

Herein, we report methods to prepare high-silica heulandite. High-silica heulandite is prepared via topotactic condensation of a layered aluminosilicate material (layered material denoted CIT-8P, condensation product denoted CIT-8) formed using an organic structure directing agent (OSDA). High-silica heulandite can also be prepared by direct synthesis in hydroxide media using an OSDA that is found to be occluded in the product (material prepared in hydroxide media denoted CIT-8H).

\section{Experimental}

\subsection{Organic structure directing agent}

The diquaternary OSDAs used in this work (shown in Fig. 1) were synthesized by reacting $200 \mathrm{mmol}$ of 1,2,4,5-tetramethylimidazole (TCI Chemicals) with $100 \mathrm{mmol}$ of dibromoalkane (Aldrich) at reflux in methanol overnight. The solvent was then removed using rotary evaporation and the product washed with ether. The product was verified using ${ }^{13} \mathrm{C}$ NMR in $\mathrm{D}_{2} \mathrm{O}$ with methanol added as an internal standard and the characterization results for the diquats are given in Table 1 . The product was ion exchanged to hydroxide form using Dowex Marathon A exchange resin and the final product concentration was determined using a MettlerToledo DL22 autotitrator using $0.01 \mathrm{M} \mathrm{HCl}$ as the titrant.

\subsection{Microporous materials synthesis}

2.2.1 Characterization. Liquid NMR spectra were recorded with a $500 \mathrm{MHz}$ spectrometer.

${ }^{13} \mathrm{C},{ }^{27} \mathrm{Al}$ and ${ }^{29} \mathrm{Si}$ solid-state NMR were performed using a Bruker DSX-500 spectrometer (11.7 T) and a Bruker 4 mm MAS

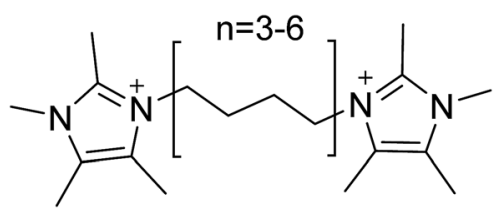

Fig. 1 Diquats of varying carbon chain linker length. probe. The spectral operating frequencies were $500.2 \mathrm{MHz}$, 125.721 MHz, 130.287 MHz and 99.325 MHz for ${ }^{1} \mathrm{H},{ }^{13} \mathrm{C},{ }^{27} \mathrm{Al}$ and ${ }^{29}$ Si nuclei, respectively. Spectra were referenced to external standards as follows: tetramethylsilane (TMS) for ${ }^{1} \mathrm{H}$ and ${ }^{29} \mathrm{Si}$, adamantane for ${ }^{13} \mathrm{C}$ as a secondary external standard relative to tetramethylsilane and $1.0 \mathrm{M} \mathrm{Al}\left(\mathrm{NO}_{3}\right)_{3}$ aqueous solution for ${ }^{27} \mathrm{Al}$. Samples were spun at $14 \mathrm{kHz}$ for ${ }^{1} \mathrm{H}$ and ${ }^{27} \mathrm{Al}$ MAS NMR and 8 $\mathrm{kHz}$ for ${ }^{13} \mathrm{C}$ and ${ }^{29} \mathrm{Si}$ MAS and CPMAS NMR experiments. For detection of ${ }^{27} \mathrm{Al}$ signal, a short $0.5 \mu \mathrm{s}-\pi / 18$ pulse was used before FID was recorded in order to make quantitative comparison among resonances.

Thermogravimetric analysis measurements were performed on Netzsch STA 449C Jupiter. Samples were heated in air to $900{ }^{\circ} \mathrm{C}$ at a rate of $1{ }^{\circ} \mathrm{C} \min ^{-1}$.

Nitrogen physical adsorption isotherms were performed at $77 \mathrm{~K}$ using a Quantachrome Autosorb iQ and the micropore volume was determined using the $t$-plot method. ${ }^{\mathbf{1 4}}$

Powder X-ray diffraction data (PXRD) data were collected on a Rigaku MiniFlex II with $\mathrm{Cu} \mathrm{K} \alpha$ radiation.

Variable temperature PXRD patterns were collected from $30{ }^{\circ} \mathrm{C}$ to $580{ }^{\circ} \mathrm{C}$ at increments of $50{ }^{\circ} \mathrm{C}$ under ambient conditions, using a PANalytical Empyrean powder diffractometer ( $\mathrm{Cu} \mathrm{K} \alpha$ radiation) equipped with an Anton Paar HTK 1200N high-temperature chamber. The sample was stabilized at each measurement temperature for 15 min before starting each measurement. The temperature ramp between two consecutive temperatures was $5{ }^{\circ} \mathrm{C} \min ^{-1}$.

Scanning electron micrographs (SEM) were recorded on a Hitachi S-570 instrument. EDS spectra were acquired with an Oxford X-Max SDD X-ray Energy Dispersive Spectrometer system on a ZEISS 1550 VP FESEM, equipped with in-lens SE.

Diffuse reflectance UV-visible (DRUV) spectra were recorded using a Cary 3G spectrophotometer equipped with a diffuse reflectance cell; zeolite samples were calcined prior to data collection.

Three-dimensional electron diffraction data were collected using the rotation electron diffraction (RED) technique. ${ }^{15,16}$ The RED software was installed on a JEOL 2010 microscope operating at $200 \mathrm{kV}$, and data were collected over a tilt range of $\pm 50^{\circ}$ with a tilt step of $0.50^{\circ}$, the exposure time is 3 seconds per tilt step.

A general synthesis procedure for the microporous materials can be found below. In all situations where a rotating oven was used, the samples were spun at $43 \mathrm{rpm}$.

2.2.2 Fluoride-mediated reactions. Tetraethylorthosilicate, aluminum isopropoxide and titanium(Iv) butoxide (if necessary) were added to the OSDA in its hydroxide form in a Teflon Parr Reactor. The container was closed and stirred overnight to allow for complete hydrolysis. The lid was then removed, and the ethanol and appropriate amount of water were allowed to evaporate under a stream of air. Once the appropriate mass was reached, aqueous HF was added and the mixture was stirred by hand until a homogenous gel was obtained. In the syntheses with $\mathrm{H}_{2} \mathrm{O} / \mathrm{SiO}_{2}=4$, a second evaporation step was normally used. The final gel molar ratios can be found in Table 2. Seeds were then added (see section 3.1) and the autoclave was sealed and placed in a rotating oven at $175^{\circ} \mathrm{C}$. Aliquots of the material 
Table 1 Carbon NMR shifts for the diquats

\begin{tabular}{ll}
\hline Dibromoalkane & ${ }^{13} \mathrm{C}-\mathrm{NMR}\left(125 \mathrm{MHz}, \mathrm{D}_{2} \mathrm{O}\right)$ \\
\hline 1,3-Dibromopropane & $7.93,7.98,9.96,29.02,31.70$, \\
& $41.81,124.62,126.42,142.05$ \\
1,4-Dibromobutane & $7.56,9.35,21.21,25.88,30.17$, \\
& $44.14,124.60,126.00,141.89$ \\
1,5-Dibromopentane & $7.76,7.82,9.61,22.82,28.58$, \\
& $31.42,44.72,124.84,126.03,141.95$ \\
1,6-Dibromohexane & $7.88,7.95,9.72,25.42,28.88$, \\
& $31.51,44.97,124.96,126.08,142.01$
\end{tabular}

were taken periodically by first quenching the reactor in water and then removing enough material for PXRD.

2.2.3 Hydroxide-mediated reactions. Molar ratios used in all hydroxide mediated reactions are given in Table 3. In these syntheses sodium aluminate (Pfaltz \& Bauer) was combined with the OSDA in hydroxide form, $1 \mathrm{M} \mathrm{NaOH}$ and any necessary water and stirred until the solution cleared. Finally, Ludox AS-40 (Aldrich) was added and stirred until a homogenous gel was obtained. Seeds were added (see Table 3 ) and then the gel was heated at $160{ }^{\circ} \mathrm{C}$ in a rotating oven.

\subsection{Calcination and ammonium exchange}

All products were calcined in breathing grade air. The material was heated to $150{ }^{\circ} \mathrm{C}$ at $1{ }^{\circ} \mathrm{C} \mathrm{min}{ }^{-1}$, held for three hours, then heated to $580{ }^{\circ} \mathrm{C}$ at $1{ }^{\circ} \mathrm{C} \min ^{-1}$ and held for six hours to assure complete combustion of the organic. After calcination, materials prepared in hydroxide media were exchanged to ammonium form using $1 \mathrm{M} \mathrm{NH}_{4} \mathrm{NO}_{3}(100 \mathrm{~mL}$ of solution per gram of catalyst) at $95{ }^{\circ} \mathrm{C}$ with stirring for three hours, this was done a total of three times per sample. After ammonium exchange the materials were washed with water and dried and then calcined to proton form using the standard method.

\section{Results and discussion}

\subsection{Fluoride-mediated syntheses}

The diquaternary OSDAs investigated in this work were first screened over a range of aluminum and water contents and the results are reported in Table 2. Several different products were produced using these diquats depending on the water and aluminum content. CIT-8P was found as a product in these screening reactions along with *BEA, CIT-7, IWV, STF and STW. This competition demonstrates the weak nature of the structure direction exhibited by these diquats, and is similar to the behavior observed in our recent work reporting CIT-7. ${ }^{17}$ Additionally, many of these products have been reported before with other diquaternary OSDAs in fluoride media, such as *BEA and STF. ${ }^{18}$ CIT-8P forms in reactions with relatively high amounts of aluminum $(\mathrm{Si} / \mathrm{Al}=15,20)$, and decreasing the aluminum content generally led to the formation of CIT-7, IWV or STF. Seeding these higher-silica syntheses with CIT-8P materials was not successful in eliminating the formation of competing phases. Therefore, CIT- $8 \mathrm{P}$ could only be formed from a limited range of synthesis compositions. Synthesis times to form CIT-8P were around 12 days without seeds added, and around 5 days when they were added (some variation in these times was observed in different synthesis trials). Phase competition was also observed, and once a pure-phase sample of CIT-8P was obtained, seeds were used in all subsequent reactions to help favor the formation of CIT-8P.

The PXRD of CIT-8P is shown in Fig. 2 along with the product that results from calcination (identified as a HEU-type material). A framework also formed by topotactic condensation similar to HEU, RRO, has been previously reported as RUB-39 for the layered precursor and RUB-41 for the calcined framework (RRO). ${ }^{19,20}$ RUB-41 is formed from heulandite-type layers and has a similar PXRD pattern as HEU. However, careful analysis of the diffraction patterns for the two materials shows distinct reflections for each of the materials. For HEU, there are strong reflections near $17^{\circ}$ and $22^{\circ} 2 \theta$ that are not present in RRO. The calcined material had a micropore volume of $0.10 \mathrm{~cm}^{3}$ $\mathrm{g}^{-1}$ from nitrogen adsorption (Fig. 4). SEM images of the asmade and calcined material are shown in Fig. 9. Both the asmade and calcined materials exhibit a plate-like morphology, similar to what has been observed in other materials prepared via topotactic condensation. EDS analysis of the calcined material found a $\mathrm{Si} / \mathrm{Al}$ ratio of $9.8 \pm 0.7$. Since the starting gel had a $\mathrm{Si} / \mathrm{Al}=20$, a large portion of the silica initially present was not incorporated in the final product. It is evident from ${ }^{13} \mathrm{C}$ CPMAS NMR that the organic is occluded intact in as-made CIT-8P (Fig. 5). The state of the aluminium in CIT-8P and CIT-8 was studied using ${ }^{27} \mathrm{Al}$ NMR and the spectra are shown in Fig. 3. In CIT-8P, only tetrahedral aluminium was observed. Calcination caused the formation of some octahedral aluminium (near $0 \mathrm{ppm}$ ). Deconvolution of the spectra shows that the calcined material contains $c a .21 \%$ octahedral aluminium.

\subsection{Mechanism of topotactic condensation}

Topotactic condensations can occur with layered materials that contain terminal silanol groups (coordination of $\mathrm{Si}(\mathrm{OSi})_{3}(\mathrm{OH})$,

Table 2 Synthesis results in fluoride media

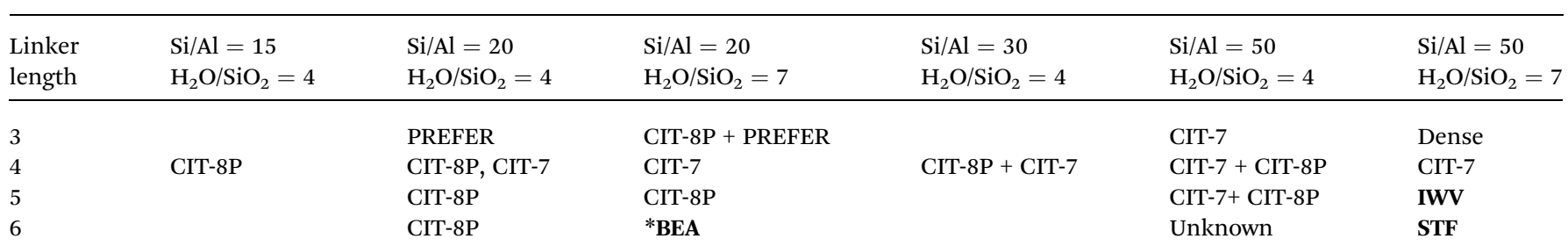


Table 3 Synthesis results in hydroxide media using the 4-carbon diquat

\begin{tabular}{|c|c|c|c|c|c|c|c|}
\hline Gel Si/Al & $\mathrm{Gel} \mathrm{Na/Si}$ & Gel ROH/Si & $\mathrm{Gel} \mathrm{H}_{2} \mathrm{O} / \mathrm{Si}$ & Temp $\left({ }^{\circ} \mathrm{C}\right)$ & Seeds & Time (days) & Product \\
\hline 4.15 & 0.25 & 0.16 & 30 & 160 & None & 51 & CIT-8H \\
\hline 5 & 0.25 & 0.16 & 30 & 160 & None & 43 & CIT-8H \\
\hline 5 & 0.25 & 0.16 & 30 & 160 & CIT-8H & 24 & CIT-8H \\
\hline 7.5 & 0.25 & 0.16 & 30 & 160 & CIT- $8 \mathrm{H}$ & 20 & CIT-7 \\
\hline 10 & 0.25 & 0.16 & 30 & 160 & CIT-8H & 20 & CIT-7 \\
\hline
\end{tabular}

$\mathrm{Q}^{3} \mathrm{Si}$ ). With calcination, these terminal silanol groups condense, releasing water and forming $\mathrm{Si}-\mathrm{O}-\mathrm{Si}$ bonds. In this process, a 2-dimensional material is converted into a 3-

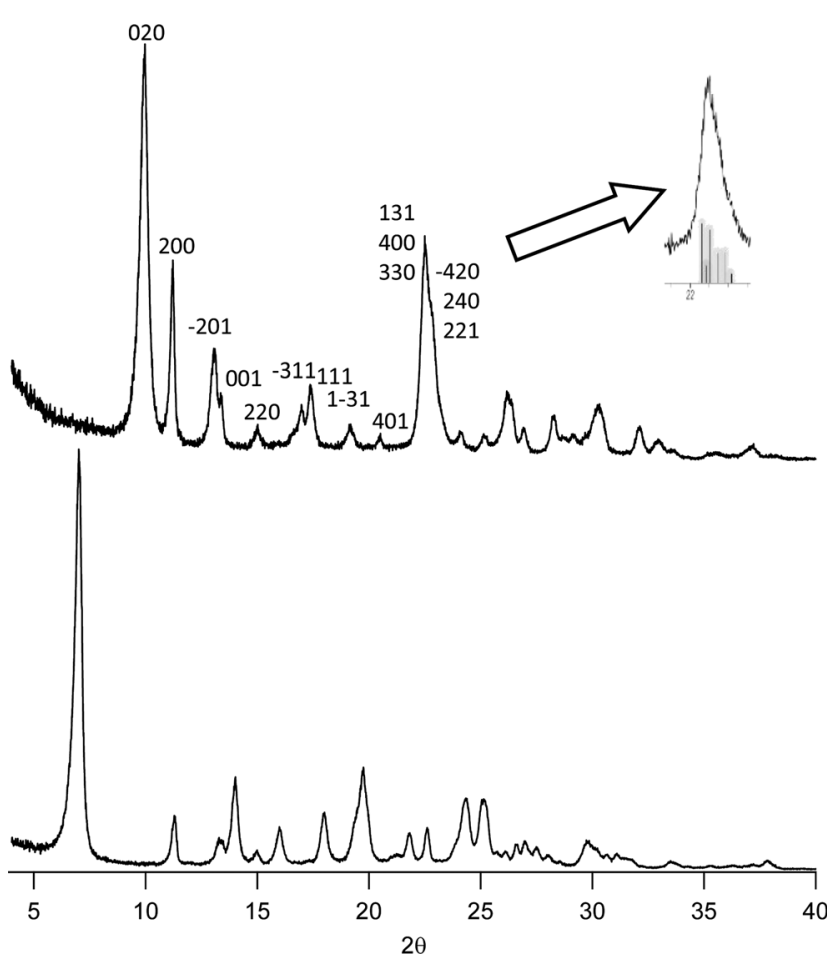

Fig. 2 PXRD of CIT-8P (lower) and CIT-8 (upper) prepared via topotactic condensation. The upper trace contains the crystallographic directions of some of the main peaks and the arrow points to a deconvolution of the prominent peak located near $22^{\circ} 2 \theta$.

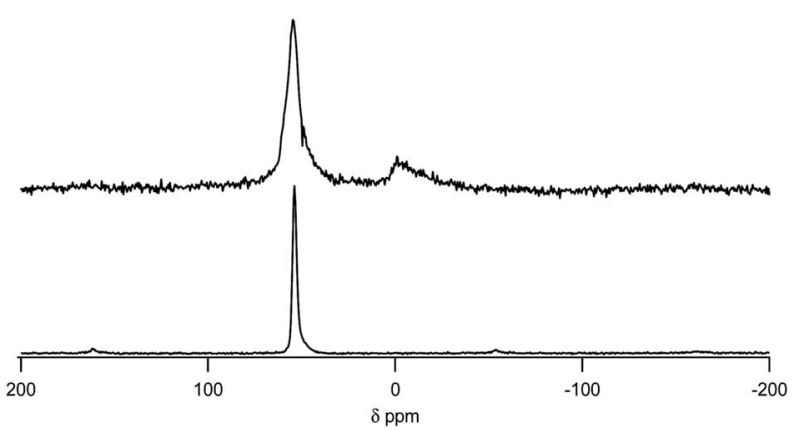

Fig. $3{ }^{27} \mathrm{Al}$ MAS NMR of CIT-8P (lower) and CIT-8 prepared by calcination to $580{ }^{\circ} \mathrm{C}$ (upper). dimensional framework material. The presence of silanol groups in CIT-8P was investigated by using ${ }^{29} \mathrm{Si}$ MAS and CPMAS NMR of the as-made and calcined materials. ${ }^{29}$ Si CPMAS NMR was used on the as-made material in addition to MAS NMR to confirm the resonances (due poor signal to noise ratio with MAS NMR often found prior to calcination). The ${ }^{29} \mathrm{Si}$ NMR spectra are shown in Fig. 7. In the calcined material, three resonances can be found at $-113.8,-108.2$ and $-102.7 \mathrm{ppm}$. In accordance

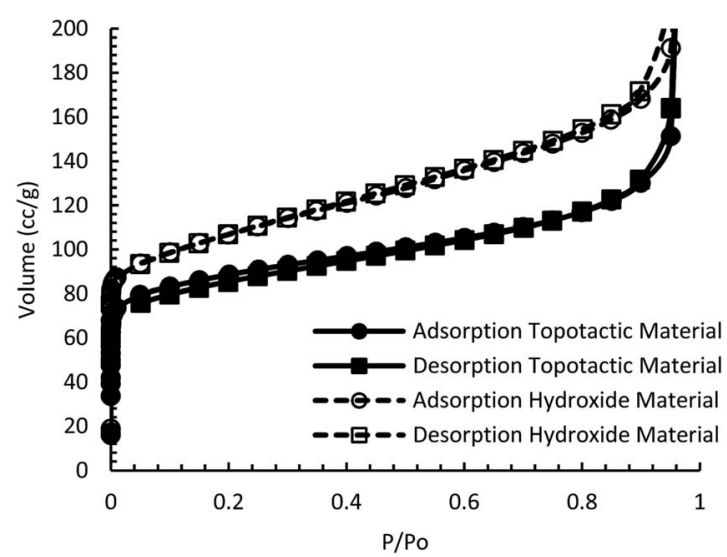

Fig. 4 Nitrogen isotherms for proton form CIT-8 and CIT-8H.

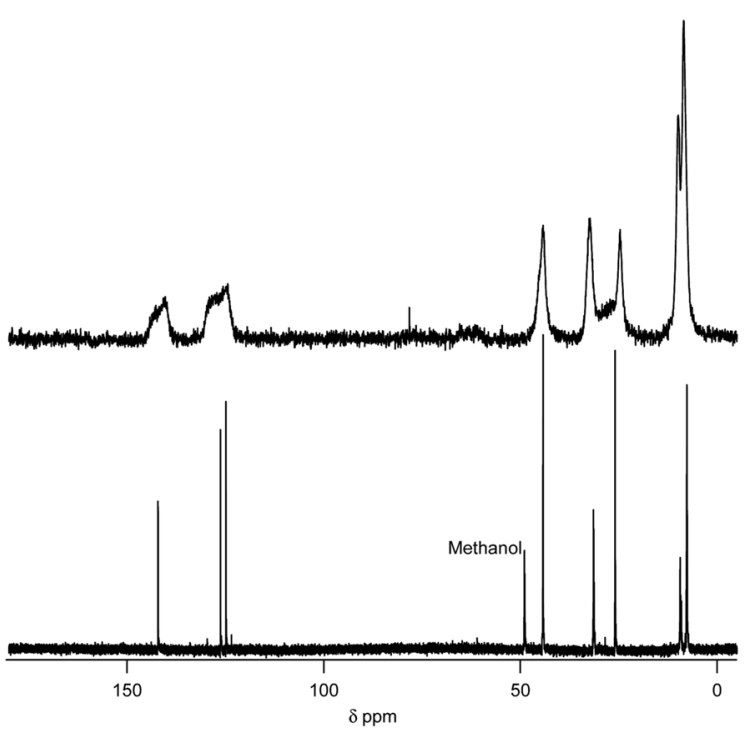

Fig. $5{ }^{13} \mathrm{C}$ liquid NMR of the diquat with a 5 -carbon chain (lower) compared to the ${ }^{13} \mathrm{C}$ CPMAS NMR of as-made CIT-8P (upper). 


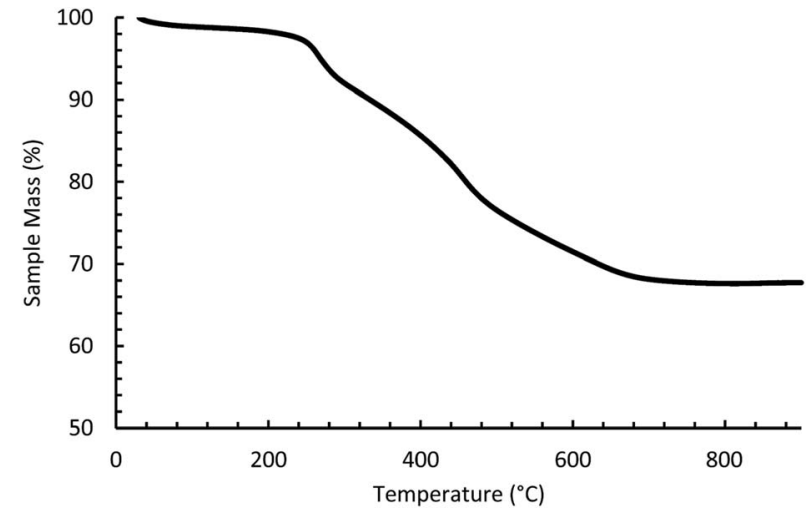

Fig. 6 TGA of CIT-8P (30.5 wt\% mass loss due to organic and fluoride).

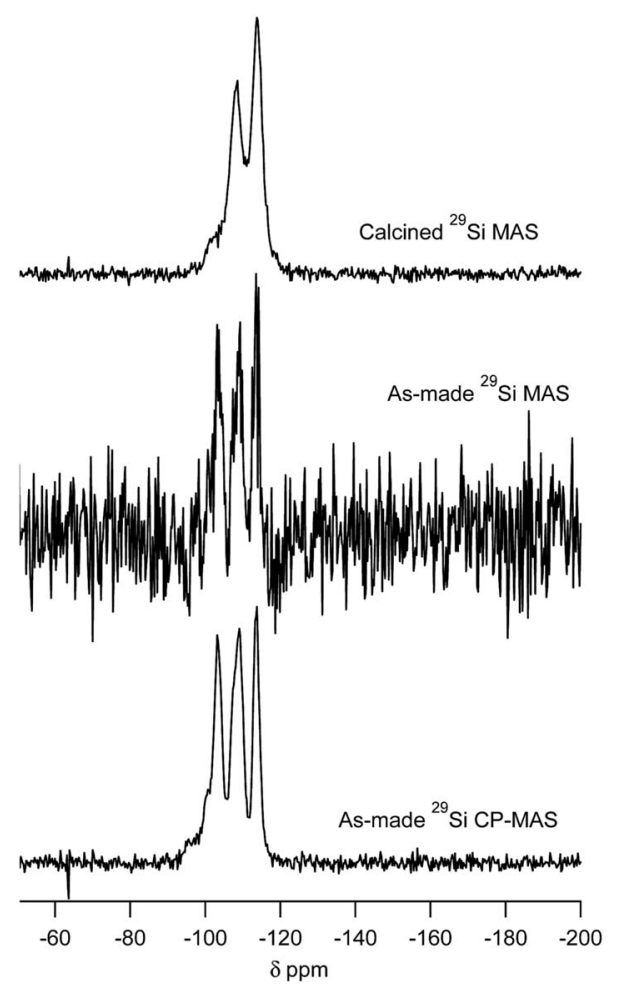

Fig. $7{ }^{29} \mathrm{Si}$ NMRs of CIT-8P by CPMAS (lower), MAS NMR (middle) compared to the calcined material (upper). with the resonances reported for other heulandite materials, the peak at $-\mathbf{1 1 3 . 8} \mathrm{ppm}$ is assigned to $\mathrm{Si}(\mathrm{OSi})_{4}$ coordination environment, and the peaks at -108.2 and -102.7 ppm are assigned to $\mathrm{Si}(\mathrm{OAl})(\mathrm{OSi})_{3}$ coordination environments. ${ }^{21-24}$ The amount of $\mathrm{Si}(\mathrm{OAl})_{2}(\mathrm{OSi})_{2}$ coordination present is very small, and deconvolution determined that the amount of silicon in this coordination is insignificant (see ESI $\dagger$ ). When the ${ }^{29} \mathrm{Si}$ MAS NMR of the calcined material was deconvoluted, the $\mathrm{Si} / \mathrm{Al}$ ratio was found to be 9.8, in good agreement with the ratio found using EDS, further supporting the peak assignments (see ESI $\dagger$ ). Both the as-made and calcined materials contain a resonance near $-113.5 \mathrm{ppm}$ that is attributed to $\mathrm{Si}(\mathrm{OSi})_{4}, \mathrm{Q}^{4}$ coordination environment as well as a resonance near $-109 \mathrm{ppm}$ that is attributed to $\mathrm{Si}(\mathrm{OAl})(\mathrm{OSi})_{3}$ coordination environment. The resonance in the as-made material near -103 ppm that largely disappears upon calcination is attributed to silanol groups $\left(\mathrm{Si}(\mathrm{OSi})_{3}(\mathrm{OH})\right)$, or $\mathrm{Q}^{3}$ Si species that are abundant on the surface of the layered material as well as $\mathrm{Si}(\mathrm{OAl})(\mathrm{OSi})_{3}$ coordinated $\mathrm{Si}^{25-27}$ The resonance at $-103 \mathrm{ppm}$ decreases significantly after calcination as the topotactic condensation (vide infra) causes the materials to loose surface silanols and form $\mathrm{Si}-\mathrm{O}-\mathrm{Si}$ bonds. Integration of the resonances in the as-made material from the ${ }^{29} \mathrm{Si}$ MAS NMR shows that $c a .30 \%$ of the $\mathrm{Si}$ is in a $\mathrm{Q}^{3}$ environment. For the ideal case of a pure-silica layered precursor, $22.22 \%$ of the $\mathrm{Si}$ atoms should be in this environment (see $\mathrm{ESI} \dagger$ ); however, the presence of aluminum also complicates the spectrum, so finding a result close to the ideal case supports the assignments. All of the results from ${ }^{29} \mathrm{Si}$ NMR are consistent with a topotactic condensation process occurring as $\mathrm{Q}^{3} \mathrm{Si}$ disappears upon calcination.

CIT-8P was investigated by variable temperature PXRD (Fig. 10) in order to elucidate the structural changes caused by the condensation. Two main events are observed in the variable temperature PXRD. The first is that the low angle peak starts to shift to a higher angle around $280^{\circ} \mathrm{C}$. When the PXRD data are combined with the TGA results (Fig. 6), it is apparent that the observed PXRD peak shift coincides with the first mass loss. The second main event observed in the variable temperature PXRD is a dramatic change between 430 and $480{ }^{\circ} \mathrm{C}$, where the low angle peak either disappears or quickly shifts to become the main peak in HEU. In the same temperature range, the TGA results show a mass loss as the final HEU framework is formed.

The structural changes caused by the condensation reaction were elucidated by monitoring the changes of the PXRD patterns from as-made material transformed via calcination. It
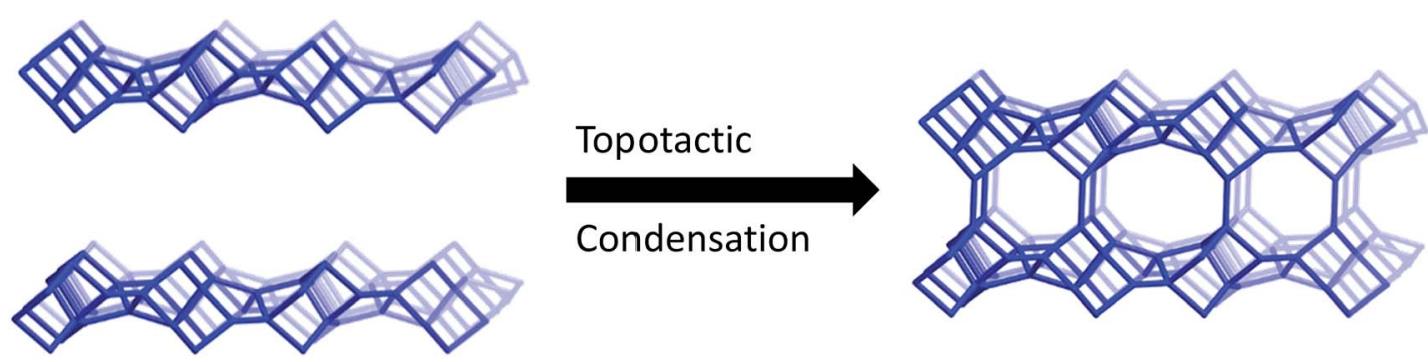

Fig. 8 Schematic representation of the topotactic condensation process. 

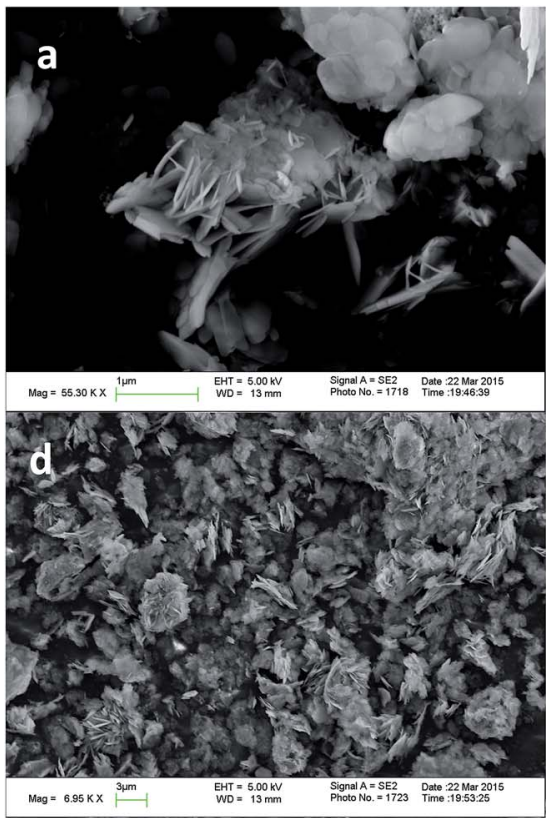

Fig. 9 SEM images: (a and b) CIT-8P, (c and d) CIT-8 and (e and f) calcined CIT-8H
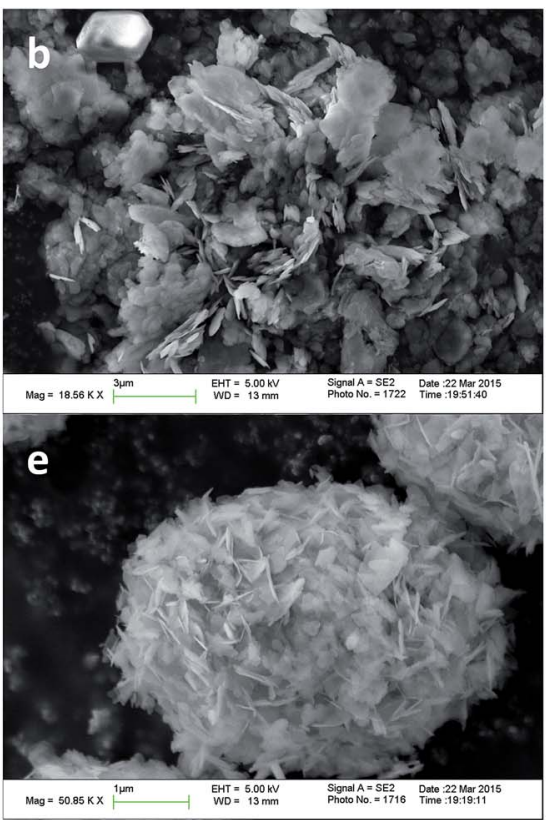

is clear from the variable-temperature PXRD that peak positions for $h 0 l$ reflections remain during heating, while the peak positions for the $h k l(k \neq 0)$ reflections are shifted to higher $2 \theta$ angles (i.e., lower $d$-spacing). This result indicates that the condensation occurs along the $b$-axis of the HEU structure and that the $a$ and $c$ axes are intact in the layered material. The asmade material was further studied using the RED technique. Three-dimensional RED tomography data (see Fig. 11) shows

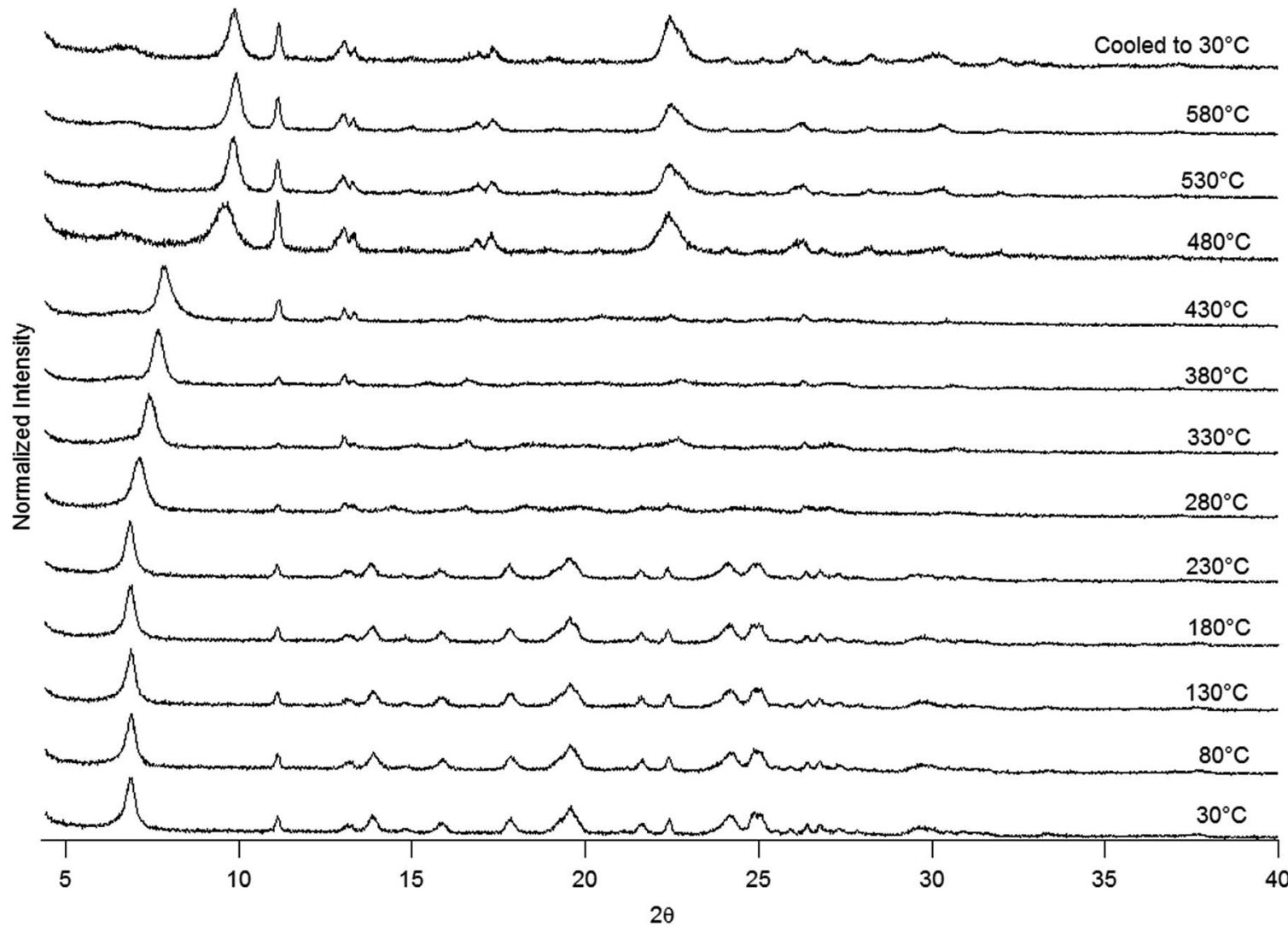

Fig. 10 Variable temperature PXRD of CIT-8P forming CIT-8. The low angle peak observed in higher traces is an instrument artifact due to the high temperature chamber. 


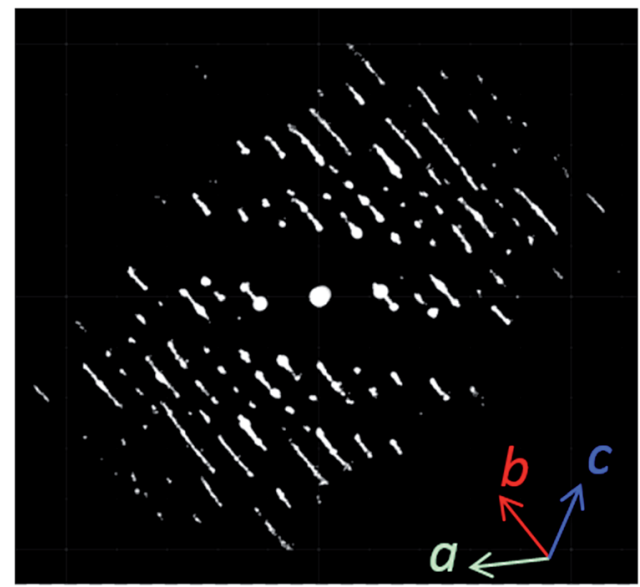

Fig. 11 RED of as-made CIT-8P

diffraction streaks between diffraction spots along $b$-axis clearly, without a significant indication of diffraction streaks along $a$ - and $c$-axes. These data indicate that in the as-made material there is some disorder in the $b$-direction, but no disorder in the $a$ - and $c$-directions, consistent with ordered inorganic layers separated by a disordered organic layer. As the material condenses along the $b$-axis, both the 10- and 8 MRs are formed (see graphical depiction shown in Fig. 8). In the as-made material, the low angle peak corresponds to an interplanar spacing of $\sim 12.8 \AA$. As the organic begins to degrade in the material (evident by TGA), the spacing is shrunk by $\sim 3.9 \AA$ until the final HEU framework is formed by topotactic condensation.

\subsection{Titanium aluminosilicate HEU}

In order to prepare a material with both Brønsted and Lewis acidity, the fluoride preparation was modified by adding titanium, with the final gel molar ratio: $1 \mathrm{SiO}_{2}$ : $0.05 \mathrm{Al}: 0.02 \mathrm{Ti}: 0.5 \mathrm{ROH}: 0.5 \mathrm{HF}: 4 \mathrm{H}_{2} \mathrm{O}$. This synthesis composition also produced the layered material CIT-8P, evident by PXRD, and the HEU framework after calcination. After calcination, the state of the titanium was studied using UV-VIS spectroscopy, and the results are shown in Fig. 12 compared to the aluminosilicate material. The band observed near $220 \mathrm{~nm}$ is consistent with tetrahedral titanium, indicating framework

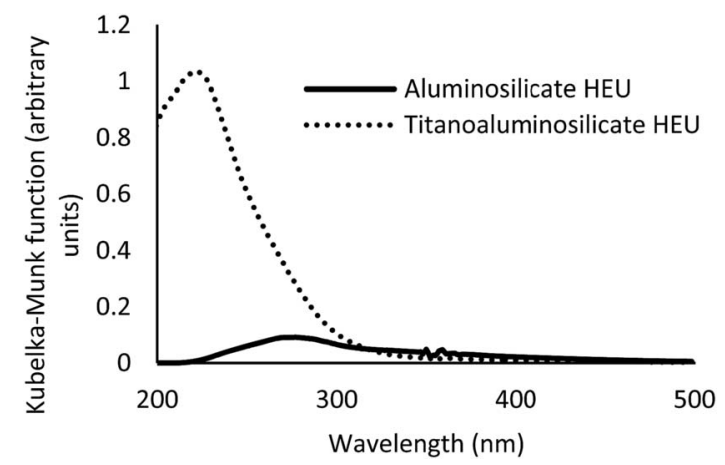

Fig. 12 UV-VIS of the calcined topotactic HEU with and without titanium.

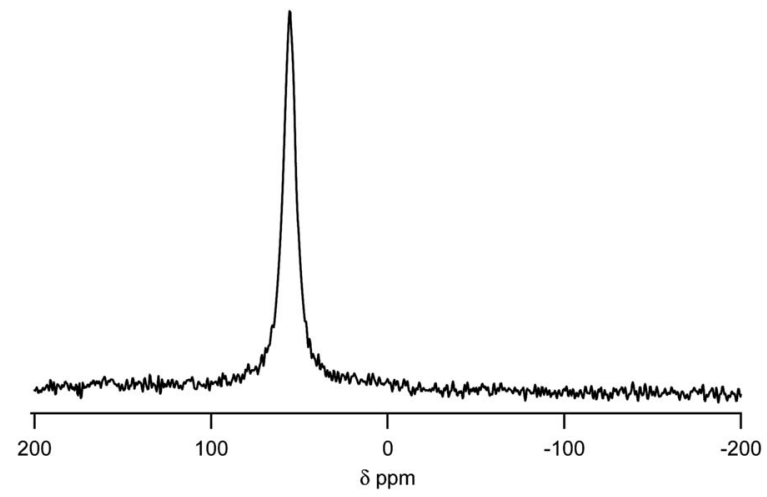

Fig. $13{ }^{27} \mathrm{Al}$ NMR of CIT-8H in proton form.

incorporation, demonstrating the synthesis of titanium aluminosilicate HEU.

\subsection{Hydroxide-mediated reactions}

The diquat containing a 4-carbon chain was used in hydroxidemediated syntheses in an attempt to produce HEU-type zeolites. Various $\mathrm{Si} / \mathrm{Al}$ ratios were tested, both with and without seeds, and the conditions and experimental results are shown in Table 3. It was found that HEU-type zeolites would not form above a gel ratio of $\mathrm{Si} / \mathrm{Al}=5$, even when seeds were present; CIT-7 was found as a product instead. At a gel ratio of both $\mathrm{Si} / \mathrm{Al}=4.15$ and 5, CIT-8H was found to form with and without seeds. Adding CIT-8H seeds at a gel $\mathrm{Si} / \mathrm{Al}=5$ significantly lowered the synthesis time from greater than 40 days to only 24 days. When seeds of CIT-7 were added at this gel ratio, CIT-7 forms instead. The CIT- $8 \mathrm{H}$ formed from a gel with $\mathrm{Si} / \mathrm{Al}=5$ (product $\mathrm{Si} / \mathrm{Al}$ ratio of $6.4 \pm 0.3$ ) was found to be stable in proton form and have a micropore volume of $0.10 \mathrm{~cm}^{3} \mathrm{~g}^{-1}$ (Fig. 4). From TGA analysis of the as-made material there was $8.4 \mathrm{wt} \%$ organic occluded that was found to be intact by ${ }^{13} \mathrm{C}$ CPMAS NMR. SEM images of the calcined material are shown in Fig. 9 and the material exhibits a plate like morphology, similar to that observed for the material prepared via topotactic condensation. The ${ }^{27} \mathrm{Al}$ MAS NMR of the material in proton form (Fig. 13) showed that all of the aluminium was in tetrahedral coordination, indicating framework incorporation. The material prepared in hydroxide media exhibits good thermal stability as it was stable to calcination, ammonium exchange and a subsequent calcination to convert it to proton form.

\section{Conclusions}

Diquaternary imidazolium compounds have been found to lead to a wide range of microporous material frameworks depending on the inorganic conditions. The synthesis of high-silica HEU-type zeolite (CIT-8) can occur via a topotactic condensation from a layered precursor (CIT-8P) as well as via direct synthesis in hydroxide media (CIT-8H). CIT-8 has a significantly higher $\mathrm{Si} / \mathrm{Al}$ ratio than previously reported materials with the HEU framework. The ability to prepare HEU-type zeolites at a higher silica composition and in the absence of trace impurities 
commonly encountered in natural materials opens up possibilities for use in new applications, such as catalysis. The inclusion of additional heteroatoms in the HEU framework, with $\mathrm{Ti}^{4+}$ as an example, demonstrates flexibility in the inorganic elements included in the inorganic framework and opens up this framework to additional applications, such as combined Brønsted and Lewis acidity.

\section{Acknowledgements}

We would like to thank Thomas Rea (Chevron Energy Technology Company) for collecting the RED data and Dr Stacey Zones (Chevron Energy Technology Company) for insightful discussions. Also, we would like to thank Dr Sonjong Hwang (Caltech) for assistance with solid-state NMR collection and interpretation. Chevron Energy Technology Company provided funding for this work. J.E.S. would like to thank the NDSEG for their support through a fellowship. PQ Corporation is thanked for providing us with sodium silicate.

\section{Notes and references}

1 C. Colella and W. S. Wise, Microporous Mesoporous Mater., 2014, 189, 4-10.

2 B. Yilmaz and U. Müller, Top. Catal., 2009, 52, 888-895.

3 G. Gottardi and E. Galli, Natural zeolites, Springer-Verlag, 1985.

4 Three-letter framework type codes (boldface capital letters) for all zeolites mentioned in the text are given in parentheses.

5 R. Simancas, D. Dari, N. Velamazán, M. T. Navarro, A. Cantín, J. L. Jordá, G. Sastre, A. Corma and F. Rey, Science, 2010, 330, 1219-1222.

6 C. Baerlocher and L. B. Mccusker, Database of Zeolite Structures, http:/www.iza-structure.org/databases/.

7 D. Zhao, K. Cleare, C. Oliver, C. Ingram, D. Cook, R. Szostak and L. Kevan, Microporous Mesoporous Mater., 1998, 21, 371379.

8 F. Related, A. Data and P. E. J. Meros, US Pat. 4,503,023, 1985. 9 O. Chiyoda and M. E. Davis, Microporous Mesoporous Mater., 1999, 32, 257-264.
10 S. Satokawa and K. Itabashi, Microporous Mater., 1997, 8, 4955.

11 C. D. Williams, Chem. Commun., 1997, 2113-2114.

12 D. Zhao, L. Kevan and R. Szostak, Zeolites, 1997, 19, 366-369.

13 A. Jayaraman, A. J. Hernandez-maldonado, R. T. Yang, D. Chinn, C. L. Munson and D. H. Mohr, Chem. Eng. Sci., 2004, 59, 2407-2417.

14 P. E. Hathaway and M. E. Davis, Catal. Lett., 1990, 5, 333-347. 15 D. Zhang, P. Oleynikov, S. Hovmöller and X. Zou, Z. Kristallogr., 2010, 225, 94-102.

16 W. Wan, J. Sun, J. Su, S. Hovmöller and X. Zou, J. Appl. Crystallogr., 2013, 46, 1863-1873.

17 J. E. Schmidt, D. Xie, T. Rea and M. E. Davis, Chem. Sci., 2015, 6, 1728-1734.

18 A. Jackowski, S. I. Zones, S.-J. Hwang and A. W. Burton, J. Am. Chem. Soc., 2009, 131, 1092-1100.

19 Y. X. Wang, H. Gies, B. Marler and U. Müller, Chem. Mater., 2005, 17, 43-49.

20 H. Gies, U. Müller, B. Yilmaz, T. Tatsumi, B. Xie, F. Xiao, X. Bao, W. Zhang and D. De Vos, Chem. Mater., 2011, 23, 2545-2554.

21 A. Godelitsas, D. Charistos, A. Tsipis, C. Tsipis, A. Filippidis, C. Triantafyllidis, G. Manos and D. Siapkas, Chemistry, 2001, 7, 3705-3721.

22 A. Godelitsas, D. Charistos, C. Tsipis, P. Misaelides, A. Filippidis and M. Schindler, Microporous Mesoporous Mater., 2003, 61, 69-77.

23 R. L. Ward and H. L. McKague, J. Phys. Chem., 1994, 98, 1232-1237.

24 E. Lippmaa, M. Magi, A. Samoson, M. Tarmak and G. Engelbardtlb, J. Am. Chem. Soc., 1981, 103, 4992-4996.

25 B. Yang, H.-H. Wu and P. Wu, J. Phys. Chem. C, 2014, 140929194213007.

26 J. Ruan, P. Wu, B. Slater, Z. Zhao, L. Wu and O. Terasaki, Chem. Mater., 2009, 21, 2904-2911.

27 P. Wu, J. Ruan, L. Wang, L. Wu, Y. Wang, Y. Liu, W. Fan, M. He, O. Terasaki and T. Tatsumi, J. Am. Chem. Soc., 2008, 130, 8178-8187. 